\title{
Bell Inequalities with Schrödinger Cats
}

\author{
M. D. Reid \\ Physics Department, University of Queensland, Brisbane, Australia \\ Reprint requests to Dr. M. D. R.; E-mail: margaret@physics.uq.edu.au
}

Z. Naturforsch. 56 a, 220-223 (2001); received February 3, 2001

Presented at the 3rd Workshop on Mysteries, Puzzles and Paradoxes in Quantum Mechanics, Gargnano, Italy, September 17 - 23, 2000.

In the Schrodinger cat gedanken experiment a "cat" is in a quantum superposition of two macroscopically distinct states. There is the apparent interpretation that the "cat" is not in one state or the other, "alive" or "dead". Here this interpretation is proved objectively. I propose the following definition of macroscopic reality: first, that the "cat" is either dead or alive, the measurement revealing which; second, that measurements on other "cats" some distance away cannot induce the macroscopic change, "dead" to "alive" and vice versa, to the "cat". The predictions of quantum mechanics are shown to be incompatible with this premise.

Key words: Bell Inequalities; Schrödinger Cats.

There has been recent experimental evidence for the generation [1] of a "Schrödinger cat state" [2], namely a quantum superposition of two macroscopically distinct states. Such superpositions are of interest, because of the paradoxical interpretation quantum mechanics apparently puts on them: that a macroscopic object (a cat) was not actually in one of two macroscopically distinct states (dead or alive), prior to its measurement. The important issue is not simply the existence of the macroscopic superposition state, but its interpretation. So far evidence, presented within the framework of quantum mechanics, has been for the existence of these states. The fundamental issue that there could be an alternative theory or interpretation of quantum mechanics, in which the "cat" is either "alive" or "dead", the measurement indicating which, is not addressed. Here I show that the predictions of quantum mechanics can be incompatible with such alternative theories. In doing so, I show that certain quantum mechanical "Schrödinger cats" irrefutably defy macroscopic reality.

Schrödinger [2] discussed the existence and interpretation of the quantum superposition of two macroscopically distinct states in his famous Schrodingercat gedanken experiment. According to quantum mechanics a particle can be predicted to be in a quantum superposition, $|\phi\rangle=(|+\rangle+|-\rangle) / \sqrt{2}$, of having escaped the nucleus, $|+\rangle$, or otherwise, $|-\rangle$. Suppose the presence of the particle outside the nucleus will trigger a lethal device that will kill a cat located in a box. An observer later examines the box to determine the state of the cat, whether dead or alive. The application of quantum mechanics, to describe the sequence of interactions, would predict the cat to be in a superposition of a state $|1\rangle$, where the cat is dead and a state $|-1\rangle$, where the cat is alive.

The predictions of the quantum superposition $(|+\rangle+$ $|-\rangle) / \sqrt{2}$ are not identical, at least for some types of measurements, to those of the classical mixture, where the system is considered to be in state $|+\rangle$ with probability $1 / 2$, or in state $|-\rangle$ with probability $1 / 2$. Yet to say in this case that the cat cannot be considered to be dead or alive prior to its measurement, here the observer opening the box to view the state of the cat, would seem nonsensical.

To establish the conclusive existence of a "Schrödinger's cat", we first propose to consider what is meant by macroscopic realism. In considering a macroscopic system (the cat) giving one of two macroscopically distinct outcomes (dead or alive) for that system upon measurement, I propose the following definition: first, that the macroscopic system (the cat) is actually in one of two macroscopically distinct states, dead or alive, prior to measurement; second that the measurement simply gives information as to which of the two states, dead or alive, the cat was 
in, and that other measurements performed simultaneously on other systems (potentially spatially separated from the first) cannot change the result of the measurement performed on the cat.

To date, the evidence for mesoscopic and macroscopic superposition states has been through the use of more indirect signatures established within the framework of quantum mechanics, and based on its correctness. Such claims are different to the claim of generating a "Schrödinger-cat" which is proven to defy macroscopic reality by way of a contradiction with the objective premise above.

Bell [4] in 1966 proposed to test quantum mechanics against all theories based on certain classical premises. Bell's result $[4,5]$ however applies to quantum superpositions of states only microscopically distinct. Leggett and Garg [6] have since demonstrated that the predictions of quantum mechanics are incompatible with a dual premise called macroscopic realism and macroscopic noninvasiveness of measurement. While this demonstration of this incompatibility is significant, the result still allows the cat to be either dead or alive, provided one accepts that the measurement of a macroscopic system alters its subsequent evolution.

I show now that for certain experimental situations, quantum mechanics can predict the existence of a "Schrödinger cat" that may defy the macroscopic reality premise above. I consider two simultaneous measurements made on two macroscopic systems designated cat $\mathrm{A}$ and cat $\mathrm{B}$. It is possible to perform, on each cat, one of two measurements. This could involve the use of optical filters, so we call these the "blue" measurement and the "green" measurement. For each measurement we have two possible outcomes, denoted by +1 and -1 , and these outcomes are macroscopically distinct, for both blue and green measurements, and for both cats A and B.

This situation of macroscopically distinct outcomes corresponds to that of the dead and alive results of measurement of a Schrödinger's cat. I now assume the premise above, that the cat $\mathrm{A}$ is either dead (result 1) or alive (result -1 ), for the blue measurement, immediately prior to the measurement. I introduce the hidden variable $\lambda_{\text {blue }}^{\mathrm{A}}$ to represent the predetermined nature of the cat, where $\lambda_{\text {blue }}^{\mathrm{A}}=1$ represents the cat dead and $\lambda_{\text {blue }}^{\mathrm{A}}=-1$ represents the cat alive. According to our premise, the result of the measurement is then given directly by the value assumed by the hidden variable.
Two different measurements, with macroscopically distinct results, can be performed at $\mathrm{A}$ and at $\mathrm{B}$ so that there are in total four hidden variables $\lambda_{\text {blue }}^{\mathrm{A}}$, $\lambda_{\text {green }}^{\mathrm{A}}, \lambda_{\text {blue }}^{\mathrm{B}}$ and $\lambda_{\text {green }}^{\mathrm{B}}$ each assuming a value either 1 or -1 . Substitution of all possible values shows: $-2 \leq$ $\lambda_{\text {blue }}^{\mathrm{A}} \lambda_{\text {blue }}^{\mathrm{B}}-\lambda_{\text {blue }}^{\mathrm{A}} \lambda_{\text {green }}^{\mathrm{B}}+\lambda_{\text {green }}^{\mathrm{A}} \lambda_{\text {blue }}^{\mathrm{B}}+\lambda_{\text {green }}^{\mathrm{A}} \lambda_{\text {green }}^{\mathrm{B}} \leq \overline{2}$. The prediction of the premise for the averages calculated over many experimental runs follows directly. We introduce: $E(\mathrm{~B}, \mathrm{~B})=\left\langle\lambda_{\text {blue }}^{\mathrm{A}} \lambda_{\text {blue }}^{\mathrm{B}}\right\rangle$, the expectation value for blue measurements at both $\mathrm{A}$ and $\mathrm{B}$; $E(\mathrm{~B}, \mathrm{G})=\left\langle\lambda_{\text {blue }}^{\mathrm{A}} \lambda_{\text {green }}^{\mathrm{B}}\right\rangle$, the expectation value for blue measurement at $A$ and a green measurement at $\mathrm{B}$; and so on.

$$
E=E(\mathrm{~B}, \mathrm{~B})-E(\mathrm{~B}, \mathrm{G})+E(\mathrm{G}, \mathrm{B})+E(\mathrm{G}, \mathrm{G}) \leq 2 .
$$

I now present a quantum state violating this inequality. We let each macroscopic system (cat) be a macroscopic field of fixed frequency comprised of two orthogonal polarisation directions. In quantum mechanics a field mode of a given frequency and polarisation is equivalent to a quantum harmonic oscillator, the energy being represented by the Hamiltonian $H=\hbar \omega \hat{a}^{\dagger} \hat{a}$ where $\hat{a}$ and $\hat{a}^{\dagger}$ are boson operators. We introduce two pairs of boson operators $\hat{\mathbf{a}}_{-}, \hat{\mathbf{a}}_{-}^{\dagger}$ and $\hat{\mathbf{a}}_{+}, \hat{\mathbf{a}}_{+}^{\dagger}$ for the two orthogonally polarised modes of cat $\mathrm{A}$; similarly we have $\hat{\mathbf{b}}_{-}, \hat{\mathbf{b}}_{-}^{\dagger}$ and $\hat{\mathbf{b}}_{+}, \hat{\mathbf{b}}_{+}^{\dagger}$ for $\mathrm{B}$.

On each system A and B a measurement is made with a polariser which transmits light polarised, at angle $\theta$ for $\mathrm{A}$, and $\phi$ for B. Here the "blue" measurement will correspond to the choice of polariser angle $\theta$ for $\mathrm{A}$, and $\phi$ for $\mathrm{B}$, while the green measurement corresponds to the choice $\theta^{\prime}$ for $\mathrm{A}$ and $\phi^{\prime}$ for B. Quantum mechanically the transmitted mode for $\mathrm{A}$ is represented by a new mode operator $\hat{c}_{+}=\hat{\mathbf{a}}_{+} \cos (\theta / 2)+\hat{\mathbf{a}}_{-} \sin (\theta / 2)$. The polariser reflects the light orthogonally polarised, and quantum theory represents this field mode by operator $\hat{c}_{-}=\hat{\mathbf{a}}_{+} \sin (\theta / 2)-\hat{\mathbf{a}}_{-} \cos (\theta / 2)$. We have similar definitions $\hat{d}_{+}=\hat{\mathbf{b}}_{+} \cos (\phi / 2)+\hat{\mathbf{b}}_{-} \sin (\phi / 2)$ and $\hat{d}_{-}=\hat{\mathbf{b}}_{+} \sin (\phi / 2)-\hat{\mathbf{b}}_{-} \cos (\phi / 2)$ for $\mathrm{B}$. The detection of the fields $\hat{c}_{ \pm}$allows determination of the total number $N_{+}^{\mathrm{A}}=\hat{c}_{+}^{\dagger} \hat{c}_{+}$, of particles in the up direction for A, and the total number of particles, $N_{-}^{A}=\hat{c}_{-}^{\dagger} \hat{c}_{-}$, in the down direction for $A$. We specify the outcome to be: +1 if $N_{+}^{\mathrm{A}} \geq N_{-}^{\mathrm{A}}$; or -1 if $N_{+}^{\mathrm{A}}<N_{-}^{\mathrm{A}}$. Similarly I define the measurement on cat B: $N_{+}^{\mathrm{B}}=\hat{d}_{+}^{\dagger} \hat{d}_{+}$and $N_{-}^{\mathrm{B}}=\hat{d}_{-}^{\dagger} \hat{d}_{-}$. Either the outcome is 1 , if $N_{+}^{\mathrm{B}} \geq N_{-}^{\mathrm{B}}$, or -1 , if $N_{+}^{\mathrm{B}}<N_{-}^{\mathrm{B}}$. 


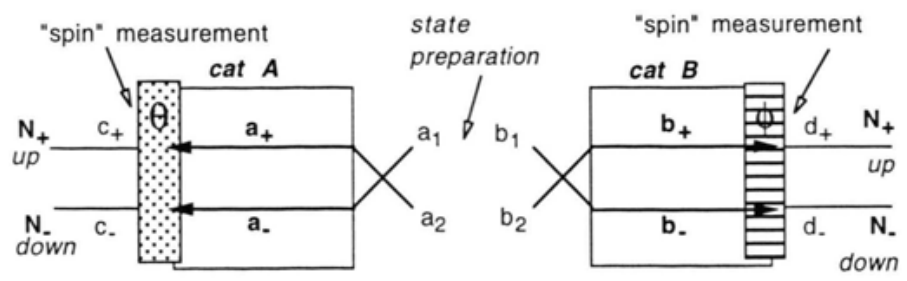

Fig. 1. Arrangement to demonstrate that Schrödinger cats A and B defy macroscopic reality.

The fields, our "cats", must be macroscopic states, and by this here we mean states of large particle or photon number. The experiment then resembles, with a macroscopic number of particles incident on the polarisers, a macroscopic version of a Bell inequality experiment [4]. While such macroscopic Bell experiments have been examined previously [7 - 12], it is an extra requirement of our experiment that the relevant outcomes for both blue and green measurements, on both cats, are macroscopically distinct, that is, correspond to macroscopically distinct states of our measured system.

We propose to generate (Figure 1) the macroscopic state for $\hat{\mathbf{a}}_{ \pm} \wedge$, and $\hat{\mathbf{b}}_{ \pm}$, from a microscopic quantum state $|\psi\rangle$ for two (harmonic oscillator) field modes designated by boson operators $\hat{a}_{1}$ and $\hat{b}_{1}$. Let [13] $\left(r_{0}=1.1\right)$

$$
|\psi\rangle=N \int_{0}^{2 \pi}\left|r_{0} e^{i \varsigma}\right\rangle_{a_{1}}\left|r_{0} e^{-i \varsigma}\right\rangle_{b_{1}} \mathrm{~d} \varsigma,
$$

where $N$ is a normalisation coefficient and $|\gamma\rangle_{k}$ is the coherent state for field mode $k$. We introduce a second pair of macroscopic quantum fields $\hat{a}_{2}$ and $\hat{b}_{2}$, in coherent states (represented in optics by laser fields) $|\alpha\rangle_{a_{2}}$ and $|\beta\rangle_{b_{2}}$ respectively, where $\alpha, \beta$ are real and large. Fields $\hat{a}_{1}, \hat{a}_{2}$ are combined (using phase shifts and semi-transparent mirrors) to give macroscopic fields $\hat{\mathbf{a}}_{-}=\left(\hat{a}_{1}-\hat{a}_{2}\right) / \sqrt{2}$ and $\hat{\mathbf{a}}_{+}=i\left(\hat{a}_{1}+\hat{a}_{2}\right) / \sqrt{2}$, incident on the polariser for A. Similarly $\hat{b}_{1}, \hat{b}_{2}$ are mixed to give macroscopic outputs $\hat{\mathbf{b}}_{ \pm}$for $\mathrm{B}$. In this way a macroscopic quantum state for the fields $\hat{\mathbf{a}}_{ \pm}$, $\hat{\mathbf{b}}_{ \pm}$is generated.

$$
\begin{aligned}
|\Psi\rangle=N \int_{0}^{2 \pi}\left|\frac{i\left(r_{0} e^{i \varsigma}+\alpha\right)}{\sqrt{2}}\right\rangle_{\hat{\mathbf{a}}_{+}}\left|\frac{\left(r_{0} e^{i \varsigma}-\alpha\right)}{\sqrt{2}}\right\rangle_{\hat{\mathbf{a}}_{-}} \\
\cdot\left|\frac{i\left(r_{0} e^{-i \varsigma}+\beta\right)}{\sqrt{2}}\right\rangle_{\hat{\mathbf{b}}_{+}}\left|\frac{\left(r_{0} e^{-i \varsigma}-\alpha\right)}{\sqrt{2}}\right\rangle_{\hat{\mathbf{b}}_{-}} \mathrm{d} \varsigma .
\end{aligned}
$$

The quantum prediction for $E$ is calculated in Fig. 2, revealing an asymptotic value of $E=2.03$ in the

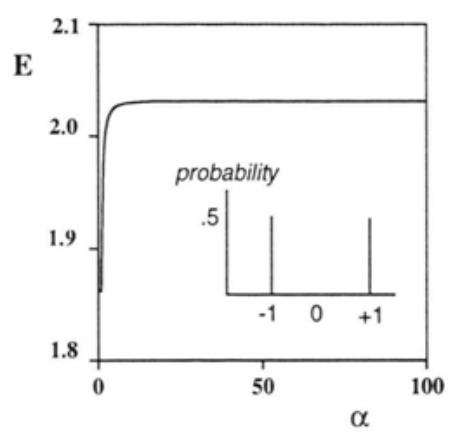

Fig. 2. Quantum prediction of $E$ versus $\alpha=\beta$, with $\theta=0, \phi=-\pi / 4, \theta^{\prime}=\pi / 2, \phi^{\prime}=-3 \pi / 4$. The inset shows the probabilities, for all choices of $\theta$ and $\phi$, of obtaining outcomes $-1,0$, and +1 , at $\mathrm{A}$ or $\mathrm{B}$, in the large $\alpha, \beta$ limit.

macroscopic limit $\alpha, \beta \rightarrow \infty$, in clear contradiction with inequality (1). The first issue is to clarify that our systems incident on the measuring apparatus at $\mathrm{A}$ and B are macroscopic. The total size of the system A is given by $N_{+}^{\mathrm{A}}+N_{-}^{\mathrm{A}}$, which as $\alpha, \beta \rightarrow \infty$ has a Poissonian probability distribution mean $\alpha^{2}$. (The fields $\hat{\mathbf{a}}_{+}$individually are macroscopic with distributions having mean $\alpha^{2} / 2$.) The next issue is to demonstrate that in this asymptotic limit, the outcomes 1 and -1 are macroscopically distinct, representing macroscopically distinct states of the system. Consider three regions of outcome: where the particle number difference $N_{+}-N_{-}$is positive and greater than $N_{0}$, result designated 1 ; where the particle number difference is less than $-N_{0} \wedge$ (result -1 ); and where the particle number is between $-N_{0}$ and $N_{0}$ (result 0 ). For $N_{0}$ macroscopic, the outcomes 1 and -1 are macroscopically distinct. For arbitrarily large, fixed $N_{0}$, the probability of obtaining a result 0 can be made an arbitrarily small value by achieving a certain ratio $\alpha / N_{0}$. As $\alpha, \beta \rightarrow \infty$, the probability of result 0 becomes negligible while the violation of our premise in this asymptotic limit is maintained, at $E=2.03$.

The violation, for (3), of an inequality similar to (1) and based on a related premise called macro- 
scopic local realism, has been discussed previously $[14,15]$. There it was shown that in the asymptotic limit: $N_{+}^{\mathrm{A}}-N_{-}^{\mathrm{A}}=\alpha \hat{X}_{\theta}^{\mathrm{A}}$ and $N_{+}^{\mathrm{B}}-N_{-}^{\mathrm{B}}=\beta \hat{X}_{\phi}^{\mathrm{B}}$, where the "quadrature phase amplitudes" $\hat{X}_{\theta}^{\mathrm{A}}$ and $\hat{X}_{\phi}^{\mathrm{B}}$ are a linear combination of the "position" and "momentum" variables of the harmonic oscillator $\hat{a}_{1}$, and $\hat{b}_{1}$, respectively. It is then possible to show that any state $|\psi\rangle$ demonstrating a failure of Bell's premise called local realism, for such continuous position and momentum variables, will violate the macroscopic realism premise we define here. Several such entangled states have been recently predicted

[1] J. R. Friedman, V. Patel, W. Chen, S. K. Tolpygo, and J. E. Lukens, Nature (London) 406, 43-45 (2000).

[2] E. Schrödinger, Naturwissenschaften 23, 807 (1935).

[3] A. O. Caldeira and A. J. Leggett, Phys. Rev. Lett. 46, 211 (1981).

[4] J. S. Bell, "Speakable and Unspeakable in Quantum Mechanics" (Cambridge Univ. Press, Cambridge, 1988).

[5] D. M. Greenbeerger, M. A. Horne, A. Shimony, and A. Zeilinger, Amer. J. Phys. 58, 1131 (1990).

[6] A. J. Leggett and A. Garg, Phys. Rev. Lett. 54, 857 (1985).

[7] N. D. Mermin, Phys. Rev. D 22, 356 (1980).

[8] P. D. Drummond, Phys. Rev. Lett. 50, 1407 (1983).

[9] S. L. Braunstein and C. M. Caves, 61, 662 (1988).

[10] A. Peres, Phys. Rev. A 46, 4413 (1992).

[11] M. D. Reid and W. J. Munro, Phys. Rev. Lett. 69, 997 (1992).

[12] N. D. Mermin, Phys. Rev. Lett. 65, 1838 (1990).

[13] G. S. Agarwal, Phys. Rev. Lett. 57, 827 (1986).

[14] M. D. Reid, Phys. Rev. Lett, 84, 2765 (2000).

[15] M. D. Reid, Phys. Rev. A. 62022110 (2000).

[16] A. Gilchrist, P. Deuar, and M. D. Reid, Phys. Rev. Lett. 80, 3169 (1998).
[16-20] and are of increasing interest, because of possible applications to quantum information and computing $[21,22]$. The photon number difference could be measured by taking the difference of two currents generated from highly efficient photodiode detectors [21], a procedure used to detect fluctuations in $\hat{X}_{\theta}^{\mathrm{A}}$ "squeezed" below the standard quantum limit. Single-mode coherent superposition states have been the subject of experimental interest [23-25]. The quantum prediction could also apply to massive particles such as bosonic atoms [26].

[17] B. Yurke, M. Hillery, and D. Stoler. Phys. Rev. A 60, 3444 (1999).

[18] A. Gilchrist, P. Deuar, and M. D. Reid, Phys. Rev. A 60, 4259 (1999).

[19] W. J. Munro and G. J. Milburn, Phys. Rev. Lett. 81, 4285 (1998).

[20] W. J. Munro, Phys. Rev. A 59, 4197 (1999).

[21] Z. Y. Ou, S. F. Pereira, H. J. Kimble, and K. C. Peng, Phys. Rev. Lett. 68, 3663 (1992).

[22] L. Vaidmann, Phys. Rev. A 49, 1473 (1994). S. Braunstein and H. J. Kimble, Phys. Rev. Lett. 80, 869 (1998). A. Furasawa, J. Sorensen, S. Braunstein, C. Fuchs, H. Kimble, and E. Polzik, Science 282, 706 (1998).

[23] C. Monroe, D. M. Meekhof, B. E. King, and D. J. Wineland. Science, 272, 1131 (1996).

[24] M. W. Noel and C. R. Stroud, Phys. Rev. Lett. 77, 1913 (1996).

[25] M. Brune, E. Hagley, J. Dreyer, X. Maitre, A. Maali, C. Wunderlich, J. M. Raimond, and S. Haroche, Phys. Rev. Lett. 77, 4887 (1996).

[26] J. Stenger, S. Inouye, D. M. Stamper-Kurn, H. J. Miesner, A. P. Chikkatur, and W. Ketterle, Nature (London) 396345 (1998). 